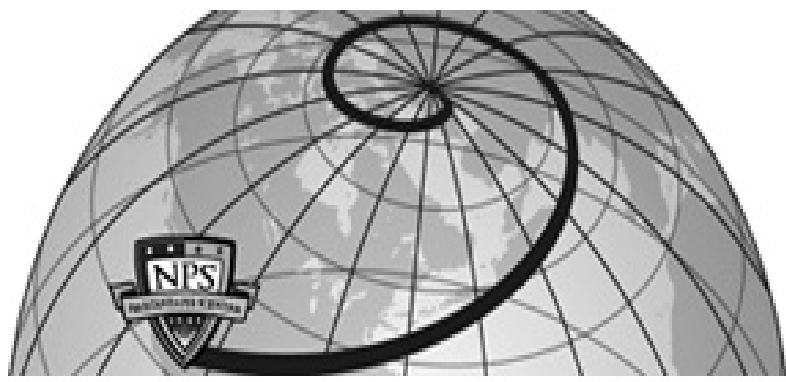

Calhoun: The NPS Institutional Archive DSpace Repository

1991-09

Spacecraft generated ions

Olsen, R.C.; Norwood, C.W.

Olsen, R.C., and C. W. Norwood, Spacecraft generated ions, Journal of Geophysical

Research, 96, 15951-15962,*1991.

https://hdl.handle.net/10945/36750

This publication is a work of the U.S. Government as defined in Title 17, United

States Code, Section 101. Copyright protection is not available for this work in the United States.

Downloaded from NPS Archive: Calhoun

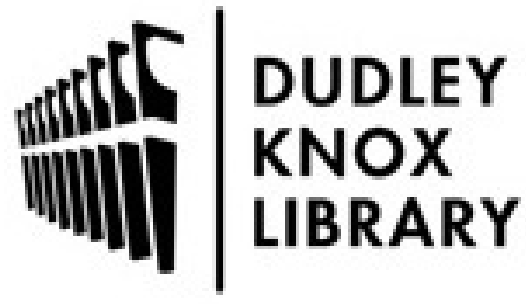

http://www.nps.edu/library
Calhoun is the Naval Postgraduate School's public access digital repository for research materials and institutional publications created by the NPS community. Calhoun is named for Professor of Mathematics Guy K. Calhoun, NPS's first appointed -- and published -- scholarly author.

Dudley Knox Library / Naval Postgraduate School 411 Dyer Road / 1 University Circle Monterey, California USA 93943 


\title{
SPACECRAFT-GENERATED IONS
}

\author{
R. C. Olsen and C. W. Norwood \\ Physics Department, Naval Postgraduate School Monterey, California
}

\begin{abstract}
Electrostatic analyzer measurements of ions on the SCATHA satellite show evidence of locally generated ions. These measurements come during periods of large negative charging ( -100 to $-10,000 \mathrm{~V})$ at or near geosynchronous altitude. Ions are observed at energies below the satellite potential, which, in the absence of scattering, must have been generated on or near the satellite surface. Differential charging measurements from the Surface Satellite Potential Monitor indicated that there were surfaces with the proper magnitude of differential charging to provide a source for the observed ions. Application of the Sigmund-Thompson theories on sputtering show that ambient $10-$ to $100-\mathrm{keV} \mathrm{O}+$ on glass should provide a yield of 0.5 to 1.0 particles per incident ion. Roughly $2-4 \%$ of this yield is ionized. This is sufficient to explain the flux levels observed. Particle tracking using the NASA Charging Analyzer Program (NASCAP) showed that the energies measured provided a sweep of trajectories along the satellite surface, but no specific source was identified.
\end{abstract}

\section{INTRODUCTION}

\subsection{Spacecraft Charging}

Spacecraft charging is the development of a potential difference between a space vehicle and its plasma environment. It is of significance for scientific purposes, since the potential may affect measurement of the ambient properties of the environment, and for practical purposes, since it may lead to anomalous command signals and physical damage to the affected spacecraft. Significant charging is most commonly found at geosynchronous orbit. The first reports of high level spacecraft charging were those for Applications Technology Satellite (ATS) 5 [Deforest, 1972]. Similar events were subsequently noted on ATS 6 and P78-2 (SCATHA) [Olsen and Purvis, 1983; Mullen et al 1986].

Generally, negative charging events are identified by a peak in the measured ion flux at an energy associated with the satellite potential, using the assumption that no ions are generated in the region of the satellite sheath. This peak in ion flux vs energy is known as the charging peak. Occasionally, ion fluxes are seen at energies below the charging peak, which would appear to violate conservation of energy for a collisionless plasma. These ion distributions have been reported previously, and tentatively ascribed to sputtering from spacecraft surfaces as ATS 5 [Deforest, 1973]. They have been termed "spacecraft generated ions", and have since been noted in observations from ATS 6 and ISEE 1. [Olsen et al, 1981; Olsen and Whipple, 1988]. At lower altitudes, sputtering by rammed, neutral $\mathrm{N}_{2}$ and $\mathrm{O}_{2}$ was observed on Atmospheric Explorer [Hanson et al, 1981].

Analysis of this particular phenomenon is of practical interest, as these ions may contaminate measurements of the ambient plasma, or the charging peak, leading to an underestimation of the satellite potential. The contamination of the charging peak may be particularly important for active experiments in low Earth orbit, such as Spacelab. Additionally, since we believe the source to be sputtering, the flux of these particles is an indication of the damage to the satellite surface caused by the vehicle's environment. The life expectancy of precision satellite surfaces, e.g. optical surfaces, and surface coatings may be directly affected by the sputtering rate appropriate to their environment.

\subsection{SCATHA Spacecraft}

The data presented here are from the Air Force P78-2 (SCATHA) satellite. The structure of the satellite and geometry ultimately determine the interpretation of the data, and are presented first. The SCATHA satellite was launched in January 1979 to collect data for the study of Spacecraft Charging at High Altitudes (SCATHA), in a joint NASA/Department of Defense program. The satellite body was cylindrical in shape with a length and diameter of approximately 1.75 meters. Seven booms were deployed in orbit from the spacecraft to provide isolation for experiments from charging effects on the satellite surface. Figure 1 depicts the SCATHA spacecraft. The University of California, San Diego, (UCSD) charged particle detector was on the forward end, and the ion gun was on the aft end; both are described below. The outer cylinder surface was divided into three general areas; two solar arrays, one forward and one aft, and a bellyband between them to provide access panels. Additionally, sections of the surface were covered in various materials, such as Kevlar and Mylar, for use in the experiments.

The solar arrays were aluminum core with a glass $\left(\mathrm{SiO}_{2}\right)$ outer face. The bellyband panels were covered with thermal paint and second surface mirrors, and acted as waste heat radiators. The forward cylinder end was coated with gold, except for some sample patches.

The vehicle was inserted into a near-geosynchronous, elliptical orbit with a perigee of $5.5 \mathrm{R}_{\mathrm{E}}$ and an apogee of 7.7 $\mathrm{R}_{\mathrm{E}}$. The orbit period was 23.5 hours, and the inclination to the equatorial plane was $7.8^{\circ}$. The satellite axis was perpendicular to the earth-sun line, and the vehicle had a spin period of $59 \mathrm{~s}$. The satellite encountered 40-day eclipse periods in both the fall and the spring. During these seasons, the spacecraft was shadowed for approximately 1 hour per day. 
SCATHA

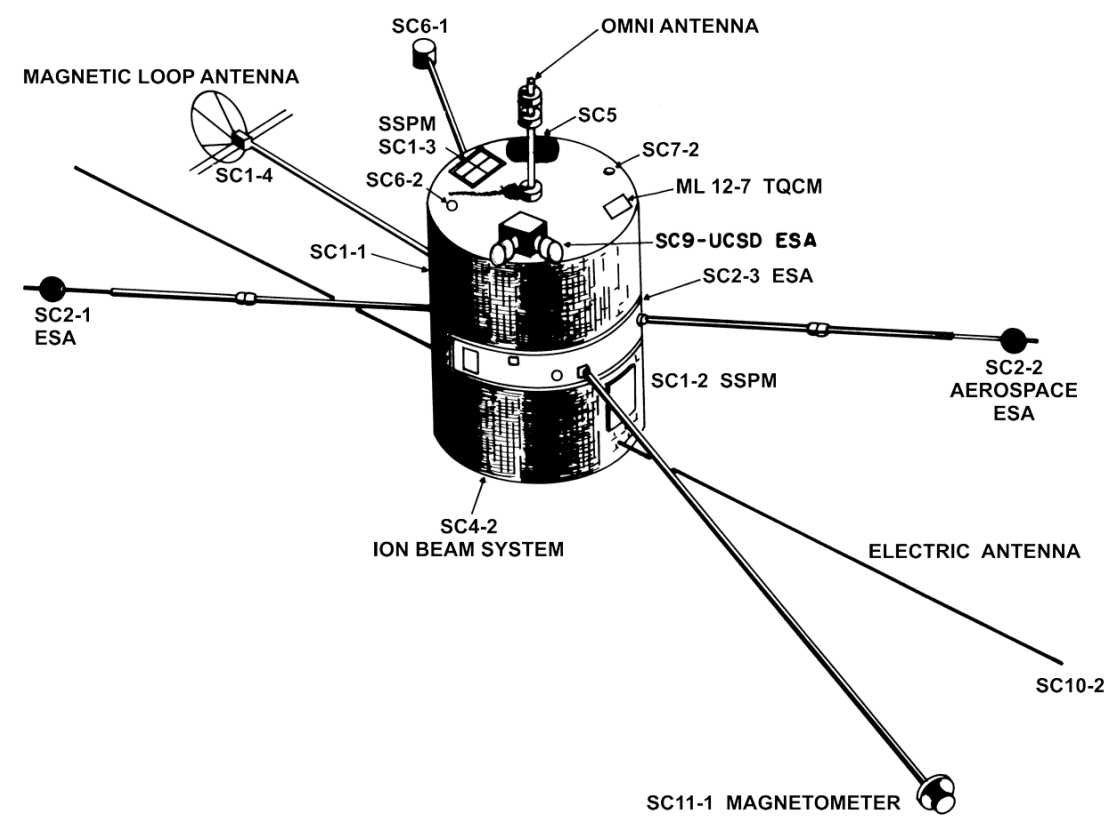

Fig. 1. SCATHA satellite, with instrument locations. The Lo detector is on the right-hand side of the instrument, the Hi detector is on the left-hand side. The Hi detector plane of rotation is almost tangential to the surface, the Lo detector plane is normal to the surface. A good photograph of the SCATHA satellite is on the cover of the December 18, 1978 issue of Aviation Week and Space Technology, (Volume 109, issue 25).

\subsection{Instruments}

The data for this paper came primarily from the UCSD auroral particles experiment (SC-9). This experiment was composed of five detectors; two pairs of rotating ion and electron detectors, and one fixed ion detector. The rotating detectors scanned in orthogonal planes and were designated the Hi set and Lo set. The Lo detector rotated from $-20^{\circ}$ to $200^{\circ}$, with $70^{\circ}$ along the spacecraft spin axis. The Hi detector scanned the same $220^{\circ}$ range, but it was more symmetrical, with its midpoint on a line parallel to the spin axis. Thus, it could not depress more than $20^{\circ}$ below the forward end plane. The fixed detector looked radially away from the spacecraft, in the same plane as the Lo detector. The detectors had an angular resolution of $5^{\circ}$ by $7^{\circ}$.

The Hi set covered an energy spectrum from $\sim 1 \mathrm{eV}$ to 81 $\mathrm{keV}$. The Lo and Fix detectors covered the 2-1800 eV energy range. For all detectors, a complete energy scan required 16 seconds and was covered in a series of 64 logarithmic steps. The energy resolution of each step was approximately $20 \%$. Additionally, the detectors could be ordered to dwell at fixed positions and/or energies.

The Lockheed mass spectrometer (SC8) provided mass and energy analysis of the $100 \mathrm{eV}$ to $32 \mathrm{keV}$ ion spectrum. This allowed for the critical element of determining the $\mathrm{H}+: \mathrm{O}+$ ratio of the incident spectrum [Quinn and Johnson, 1982].

The Aerospace Satellite Surface Potential Monitors (SSPM) provided measurements of potential for materials isolated from the satellite frame. The SSPM data indicate the level of differential charging on the insulating materials which make up the bulk of the SCATHA surface, though quantitative estimates are hard to extrapolate. [Mizera, 1981; 1983].

The Aerospace particle detector (SC2) mounted on the belly band is also utilized for this study. This detector had the capability of measuring ions from $\sim 10 \mathrm{eV}$ to $14 \mathrm{keV}$. The SC2 instrument typically sampled 21 channels in $\sim 3 \mathrm{~s}$, allowing $\sim 20$ angular measurements per spin. This good resolution of satellite spin effects neatly complements the high-energy resolution SC9 measurements [Fennell, 1982].

\subsection{Ion Gun}

An ion gun was installed on the SCATHA spacecraft to investigate the efficiency of an ion emission system in modifying satellite potentials [Masek and Cohen, 1978]. The AFGL ion gun experiment (SC4-2) was successfully used to develop negative voltages on the vehicle [Werner, 1988]. The experiment utilized xenon gas ionized by cathode discharge and accelerated by either a 1 or $2 \mathrm{kV}$ potential drop. The beam current could be varied incrementally from $0.3 \mathrm{~mA}$ to $2.0 \mathrm{~mA}$. The artificially induced negative charging episodes allowed relatively long, stable measurement periods, unlike natural daylight charging.

\section{OBSERVATIONS}

Spacecraft generated ions have previously been identified in data from ATS-5 [Deforest, 1973], ATS-6 [Olsen et al, 1981], and ISEE-1 [Olsen and Whipple, 1988]. Data from the SCATHA satellite are shown here for 2 events, one during a naturally occurring, eclipse charging period, and one for a negative charging period induced by ion gun operations. 


\subsection{Day 83 of 1979 .}

The first data set is from a naturally occurring charging event in eclipse on 24 March 1979 (Day 83). The satellite is at local midnight, $6.2 \mathrm{R}_{\mathrm{E}},-18^{\circ}$ magnetic latitude. This was a moderately active day $(\Sigma \mathrm{Kp}=21-)$, but the environment was relatively stable during the three hour period of interest $(\mathrm{Kp}=$ $2+)$. The data are presented first as a spectrogram in Plate 1. Data are shown for the Hi electron detector and ion detectors (0-81 keV), which are 'parked' viewing parallel to the satellite spin axis (roughly east). This is indicated by the line plot for the detector angle (4), between the plots for the ions and electrons. Also shown are data from the Lo and Fix ion detectors $(2 \mathrm{eV}-2$ $\mathrm{keV})$. The Lo detector is rotating over its full $220^{\circ}$ range, while the Fix detector views perpendicular to the spin axis. The view angle for the Lo detector $(\theta)$ is plotted above the Lo ion data. The charging data are in general ordered by detector angle, not pitch angle.

For the electron data, the data are plotted with energy or the vertical axis, increasing upward, and time on the horizontal axis. The flux is color coded, with purple/blue showing low flux, and red high flux. For the ion plots, the energy axis is increasing downward. The color bar is coded with the flux, using the algorithm Biased $\log =1000 \log _{10}(\mathrm{CR}+10)$, where $\mathrm{CR}$ is the count rate. The color scale has been adjusted so that ion counts below 10/s ( 2/accumulation) are plotted as white.

High electron fluxes are found up to $\sim 20 \mathrm{keV}$, which is a necessary criteria for satellite charging. Photoelectron fluxes are found at low energies in the electron data for the first 4-5 min, and the last $5 \mathrm{~min}$ of the 1-hour display. They show up as a horizontal red band at low energies. During the eclipse, (1743-1835 UT) these electron disappear, and the satellite charges negative. The satellite charges to a potential which varies around 100-200 volts negative, as indicated by the charging peaks in the ion detectors. "Shadow" peaks are found at lower energies in each detector, with varying characteristics. Two shadow peaks are visible in the Hi ion detector as roughly horizontal traces of purple or dark blue spots. These track the satellite potential from 1750-1820 UT. In the Lo ion detector there are patterns which repeat with the 5 minute period of the detector rotation. The triangular pattern has a minimum energy (vertex) coinciding with the end of the angular sweep $(\psi=$ $200^{\circ}$ ). In the Fix ion detector, a shadow peak is visible beginning at $\sim 1750$. Short vertical striations in the ambient plasma measurement are due to pitch angle modulation (fieldaligned ion distributions).

Figure 2 is a plot of the ion distribution function (phase space density) versus energy for the Hi detector. The spacecraft is charged to $-250 \mathrm{~V}$, in eclipse, as indicated by the peak in phase space density (f) at $250 \mathrm{eV}$. The shadow peak is the secondary peak at $78 \mathrm{eV}$. At this time, the detector is parked at $92^{\circ}$ detector angle, which is approximately parallel to the spacecraft spin axis (also approximately $90^{\circ}$ pitch angle).

Figure 3 is a diagram of the spacecraft potential, shadow peak energy, and Lo detector look angle versus time. The energy of the peak varies directly with the look angle, with the energy minimum occurring at the angle maximum, that is, looking down and away from the spacecraft (where it approaches closest to the spacecraft surface). The shadow peak energy maintains a relatively constant ratio with satellite potential. In this case, $E_{p} / E_{t}$ is between 6 and 7 , where $E_{p}$ is the

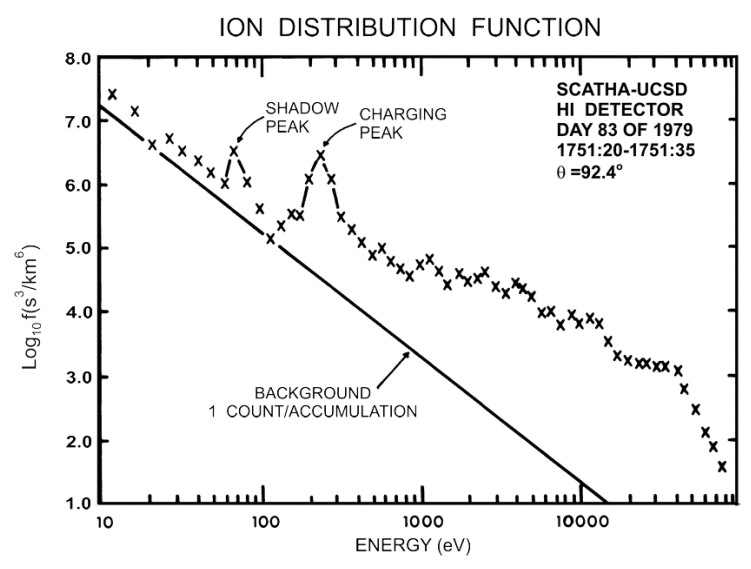

Fig. 2 lon distribution function from the $\mathrm{Hi}$ ion detector on day 83 of 1979 .

satellite potential, and $E_{t}$ is the triangle peak minimum energy. This is a consistent feature of the data, with relatively constant ratios during most charging events.

The coupled energy-angle dependence of the shadow peak is similar to the energy-angle dependence found in low-energy electron data on ATS-6. This feature was associated with a localized, differentially charged surface on the ATS-6 body (reference "Minnesota spots", Olsen et al [1981]). Each trajectory back to the emitting surface determines a unique energy and emission angle.

The SSPM data showed that for the materials which did differentially charge (gold plated magnesium, optical solar reflector (OSR, effectively a glass surface)) the charging was negative with respect to the satellite frame. Hence these measurements give no direct evidence for the surface material which is emitting the ions.

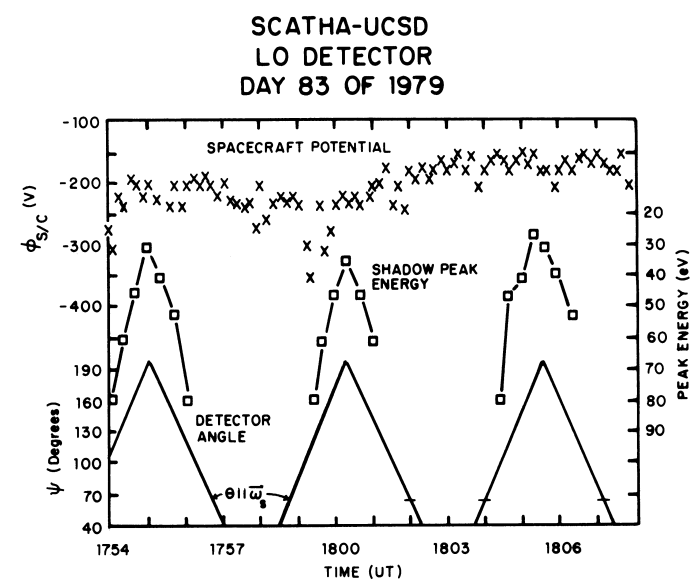

Fig 3. The spacecraft potential for a portion of the eclipse period on day 83 of 1979 is plotted in the top portion. The energy of the shadow peak in the Lo ion detector is plotted in the center, with the look direction of the Lo detector plotted at the bottom. At $68^{\circ}$ look direction, the Lo detector is viewing parallel to the spin axis. This point is indicated on the bottom panel for two times near 1757 UT. 


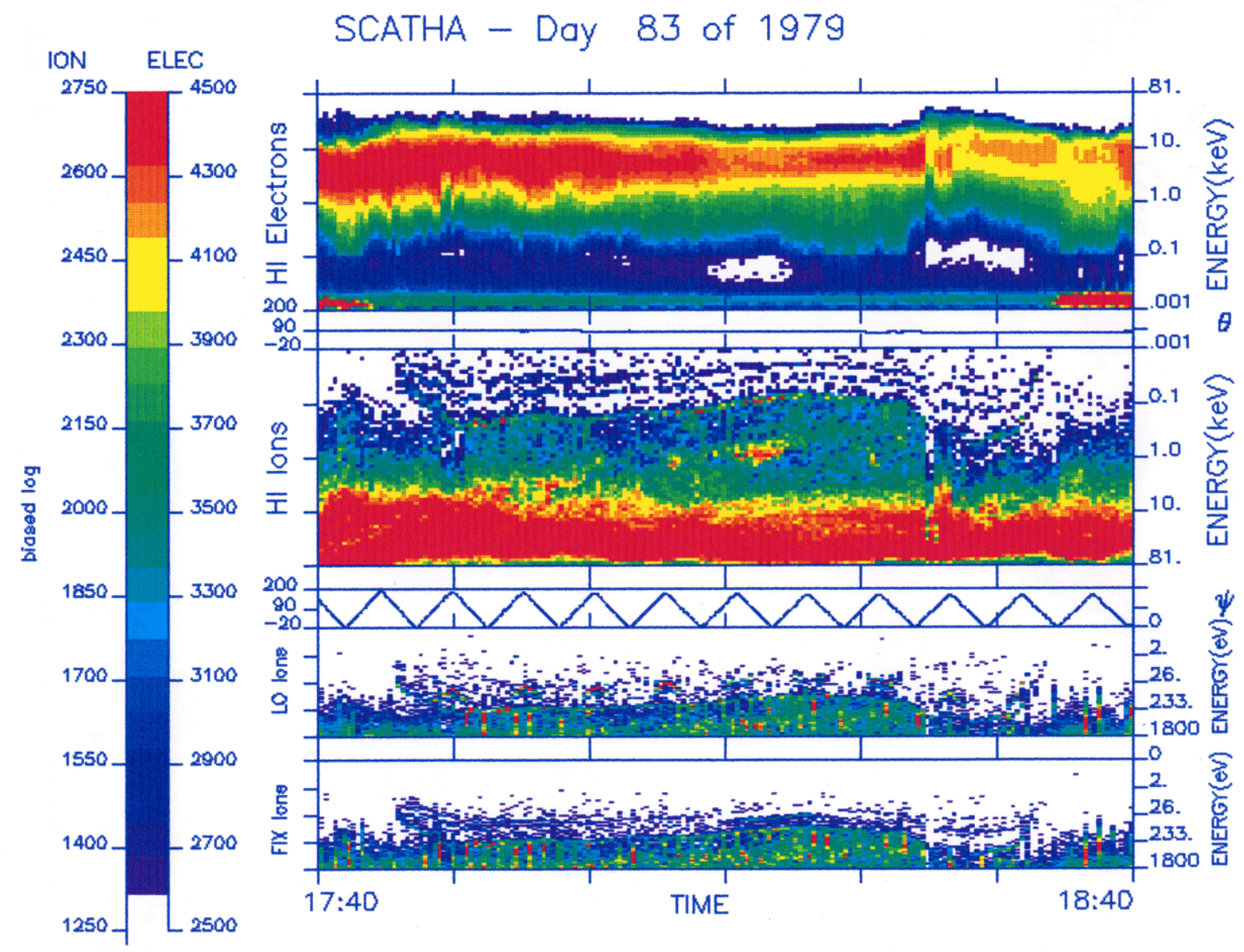

Plate 1. Spectrogram for UCSD plasma data on day 83 of 1979. The count rate (differential energy flux) is plotted as a function of time (horizontal axis) and energy (vertical axes). For electrons, the energy axis is increasing upwards. The ion data are plotted with energy increasing downwards. The coding algorithm for the color scale is to use the biased $\log$ of the count rate: $\mathrm{BLOG}=1000 \log (\mathrm{CR}+10)$. The top panel is the $\mathrm{HI}$ electron data with the associated look direction plotted below. This detector was parked viewing along the spin axis, at approximately $90^{\circ}$ pitch angle. The next color panel is the $\mathrm{HI}$ ion data. The bottom two panels are the Lo ion and Fix ion data. 


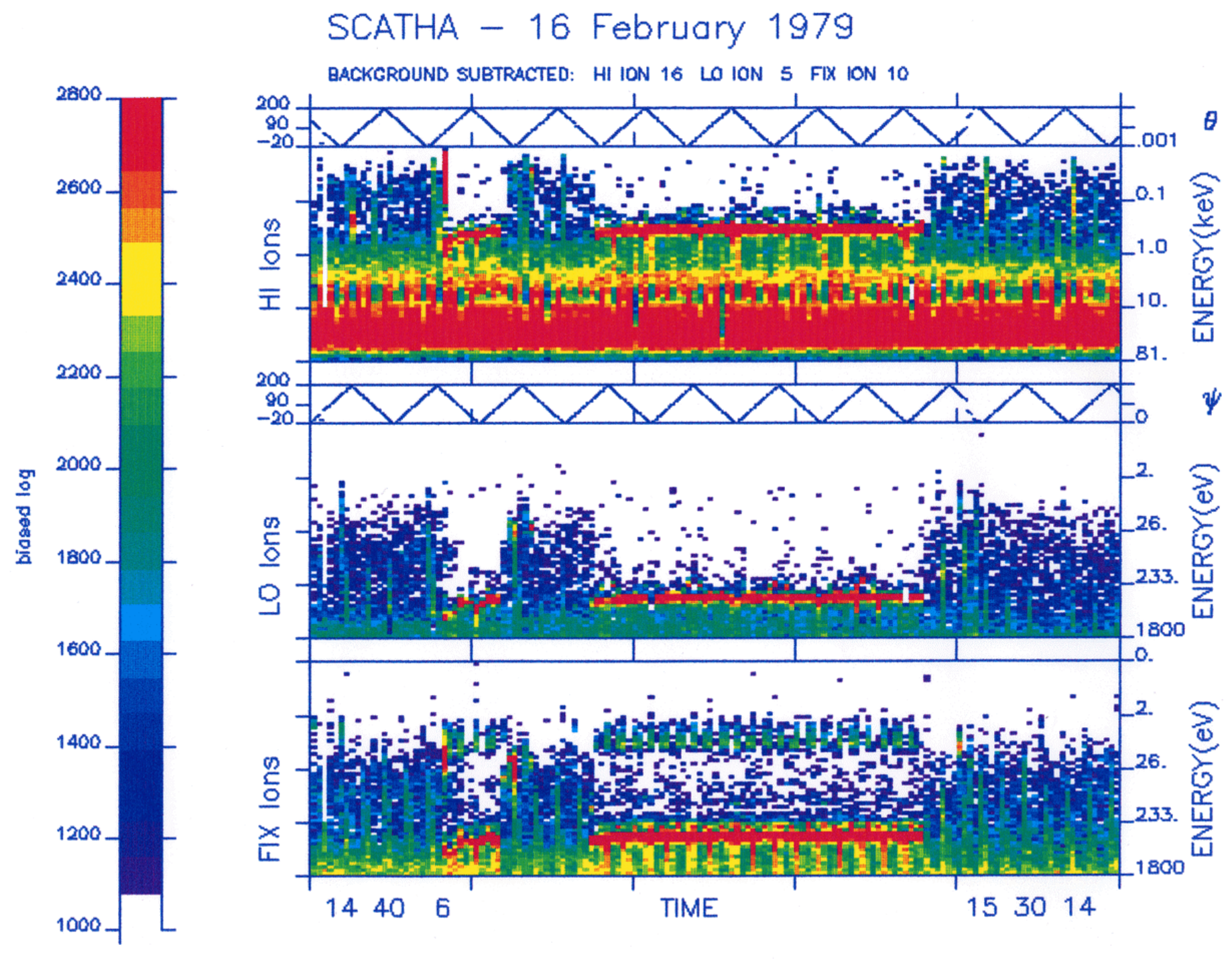

Plate 2. Spectrogram for UCSD plasma data on day 47 of 1979. Only the data from the three ion detectors are presented. 
The example illustrated here is typical of observations during eclipse passages for which negative charging occurred. By comparison with ATS 6, we would expect similar observations during daylight negative charging events. ATS 6 , however, was 3-axis stabilized and had its large dish antenna to provide a shadowed area which would simulate an eclipse. SCATHA had no such antenna, and in addition was spinning so that both the spacecraft potential and differential potentials were spin modulated. This made it difficult to use the UCSD data for this project, because the energy sweep was slow compared to the spin rate. However, induced charging events with the ion gun provide an additional range of daylight data. An example from one ion gun experiment is presented next.

\subsection{Day 47 of 1979}

The example chosen is from an ion gun experiment on 16 February 1979 (Day 47). Three operation sequences were run on Day 47. All provided similar results, indicating little or no dependence on local time (e.g. midnight, dawn, noon). The second operation, at $\sim 0730 \mathrm{LT}, \mathrm{L}=7.7$ is chosen for convenience. The ion gun was operated at $1 \mathrm{kV}$ accelerating voltage, with a nominal $1 \mathrm{~mA}$ beam current.

Plate 2 shows the UCSD particle data. The Hi and Lo detectors are rotating. The top panel shows the Hi ion data, the line plot above shows the detector angle $(\theta)$. At the low end of the angular sweep $\left(-20^{\circ}\right)$ the detector is looking away from the spacecraft. At the maximum angle $\left(220^{\circ}\right)$ the field of view may include the satellite surface (the top deck). When the ion gun is off, the satellite is positive, and the satellite potential is positive. When the gun is on, the satellite charges to $\sim-400 \mathrm{~V}$, as indicated by the nearly constant energy of the red charging peak. It is believed that the satellite does not charge to the beam energy because of space-charge effects in the beam. There are two gun operations periods illustrated; the first is at $~ 1450$ UT, the second from 1457-1517 UT. Spacecraft generated ion fluxes are visible in all three ion detectors. In the Hi and Lo detectors, the energy of the return flux is modulated by the detector rotation angle. For the $\mathrm{Hi}$ detector, the minimum energy occurs at the minimum angle $(\theta)$, while the Lo detector shows the same behavior observed previously; minimum energy occurs at maximum angle $(\psi)$

The Fix ion detector shows a different pattern. Viewing perpendicular to the spin axis, the flux is at low energies (2$10 \mathrm{eV}$ ) and is spin modulated. (The spin period early in the mission was about $50 \mathrm{~s}$.) Above the charging peak, there is a pitch angle modulation to the ambient ion data.

Figure 4 shows the UCSD ion data as $\log \mathrm{f}$ vs $\log$ energy plots. Both the $\mathrm{Hi}$ and Lo ion detectors are viewing roughly parallel to the spin axis, while the Fix detector views radially. All three detectors indicate a spacecraft potential of $\sim-370 \mathrm{~V}$. There is a second peak in the Fix ion distribution function at 6$10 \mathrm{eV}$ (also a peak in flux).

Similar results are found in the Aerospace detectors. The body mounted SC2-3 views radially outward from the bellyband. This electrostatic analyzer was operated with shorter energy sweeps, allowing better angular distributions. Figure 5 shows a grey-scale spectrogram for the SC2-3 detector. Zero flux is coded as black, high flux is white. The spin modulation is apparent in both the electrons (top) and ions (bottom). There is a charging peak in the $400 \mathrm{eV}$ channel, and a repetitive structure which looks like an inverted "V" in the ion data.

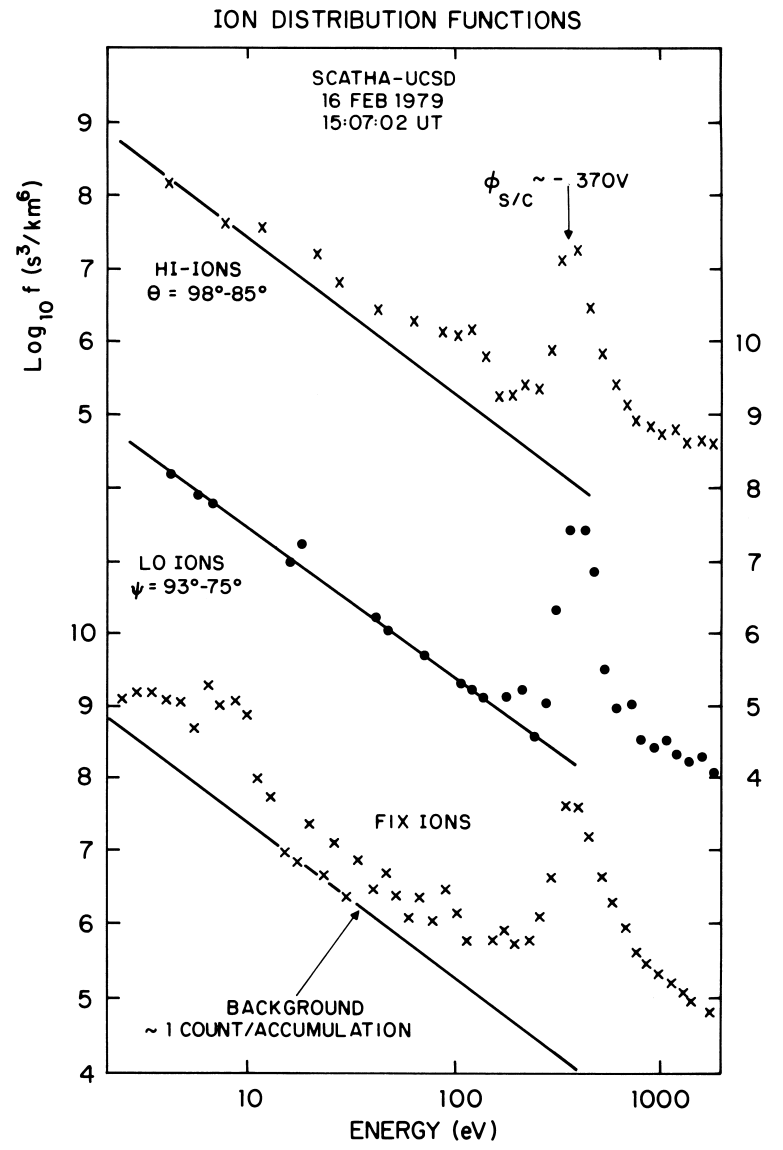

Fig. 4. Ion distribution functions for day 47 of 1979 , for the three ion detectors. Data were selected for times when the $\mathrm{Hi}$ and Lo detectors were viewing approximately along the spin axis.

Figure 6 shows the Aerospace ion data as line plots, plotted versus spin phase. It can be seen that these low energy ions are observed on the shadowed side of the spacecraft. These data can be explained if the potential of the emitting surface is varying with spin. This is reasonable, since illumination of the surfaces near the SC2-3 detector does vary with spin.

The magnitude of the spin modulation of the insulator potentials can be checked by considering the SSPM data. Figure 7 shows the potential of the large and small Kapton samples measured with respect to the satellite frame. The bottom panel shows the gold-coated magnesium. When the large Kapton sample is in sunlight, it floats $\sim+180 \mathrm{~V}$ with respect to the spacecraft. As it moves into shadow, its potential drops due to the decrease in photo electron current. The drop has a time constant which reflects the relatively large capacitance of the material. At its minimum, the potential difference reaches $\sim+100 \mathrm{~V}$. This modulation in potential of $\sim 100 \mathrm{~V}$ reflects the variation in energy of the ions observed by SC2-3. Other materials in the SSPM experiment showed spin modulations from +50 to $+100 \mathrm{~V}$ (small Kapton), and +25 to +125 (gold coated magnesium). The identity of the source surface is unknown, but it appears that the spin modulated potentials reported by the SSPM are consistent with the values inferred from the SC2-3 ion measurements, assuming these ions are emitted from differentially charged insulating surfaces. 
SCATHA SC2-3 16 FEB 79

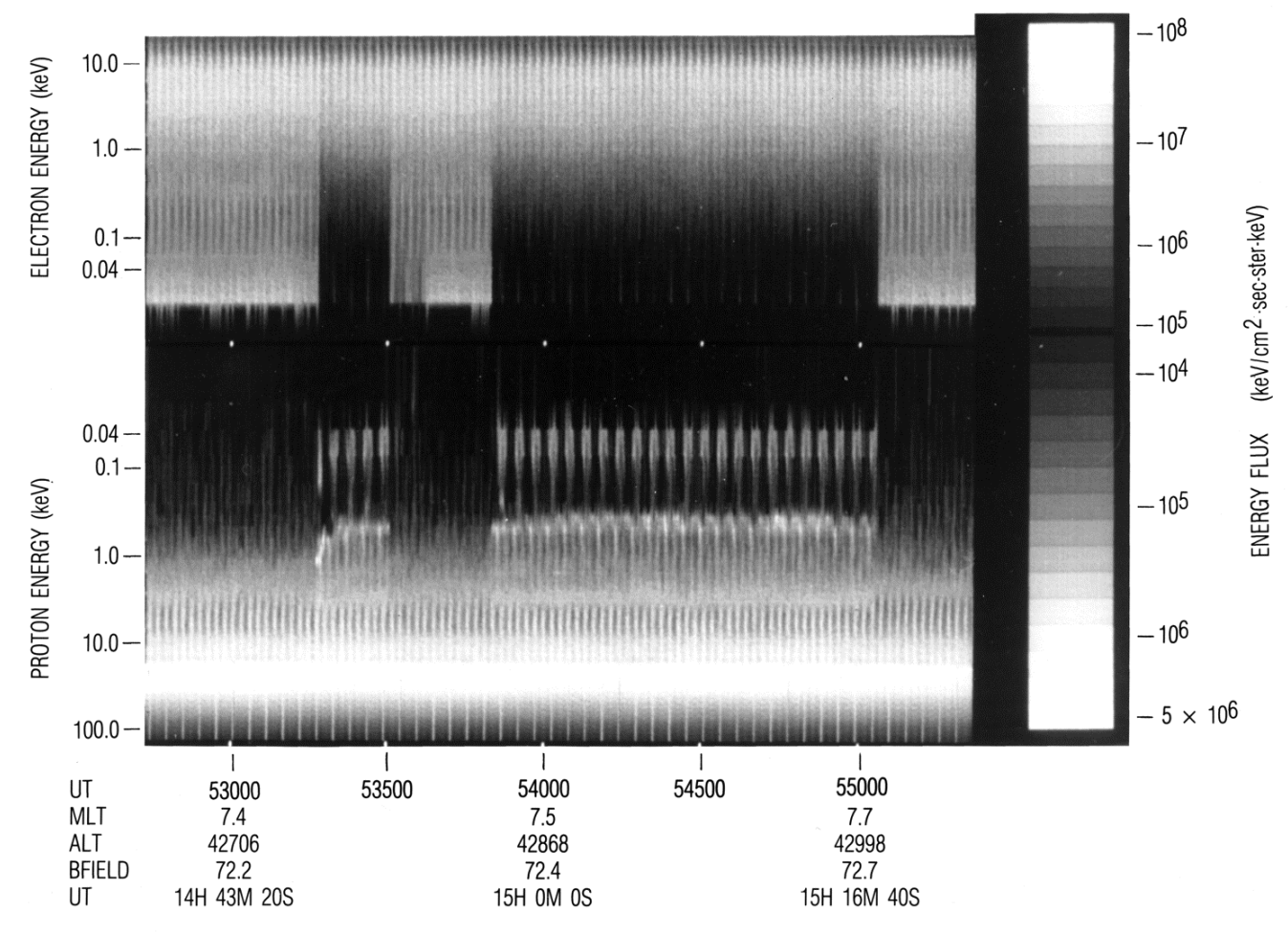

Fig. 5. Spectrogram for Aerospace plasma data on day 47 of 1979. The top panel shows electron data, the bottom panel ion data. White is used for high flux, black for zero counts. The Aerospace detector is viewing radially from the body.

\subsection{SUMMARY OF OBSERVATIONS}

Two other days were analyzed in detail, with results used below. Day 86 was similar to day 83 , with an eclipse potential of a few hundred volts negative. On day 200 there was a sequence of ion gun experiments in daylight, with results similar to those on day 47. Additional periods were analyzed by Norwood [1988].

The observations of spacecraft generated ions in eclipse and during ion gun experiments reveal two main categories. There are diffuse spectra, with a maximum flux at low energies. Examples are the measurements from the UCSD Fix detector and Aerospace detectors on day 47. The second category is more common, showing $\sim$ monoenergetic "shadow peaks" tracking the charging peak found in the UCSD detectors. This is most evident when the detectors are parked. The Hi detector data from day 83 show this feature. When the Lo detector is rotating, the "shadow peak" forms a triangular pattern, as found on day 83 .

\section{ANALYSIS}

Two possible sources for ion generation seem likely: outgassing and sputtering. The original studies of this topic by DeForest led him to believe that sputtering yields for $\mathrm{H}+$ on $\mathrm{SiO}_{2}$ were marginally sufficient to explain the observed fluxes [Deforest, 1973; private communications, 1977]. Therefore, outgassing was pursued as an additional source. Cauffman [1973], showed that gas molecules leaving the satellite would be ionized at relatively large distances. Therefore, the majority of the ionized gas would return to the satellite at energies near the satellite potential. This source apparently would not explain the variation in energy with detector angle. Also outgassing will decrease exponentially with time. Analysis of SCATHA data taken 2 years later (1981) showed that the spacecraft generated ion fluxes were unchanged [Norwood, 1988]. Outgassing may still contribute to the ion flux in the charging peak, and may explain the relatively high fluxes encountered in the ATS-6 charging peaks [Scialdone, 1986]. It remains to be shown that sputtering can explain the observed ion fluxes.

Sputtering of ions from differentially charged surfaces should explain all the major characteristics of the observations, particularly with the realization that $10-50 \%$ of the incident ions are $\mathrm{O}^{*}$. This is important because the yield for $\mathrm{O}+$ is two orders of magnitude higher than the yield for $\mathrm{H}^{*}$. The demonstration that sputtering will explain the observed results requires a model for the satellite, local potentials and trajectories, and a model for the emitted flux as a function of the incident ion distribution. Ideally, the models should be combined. This was beyond the scope of reasonable work for this project, however, and the two aspects are addressed separately. 


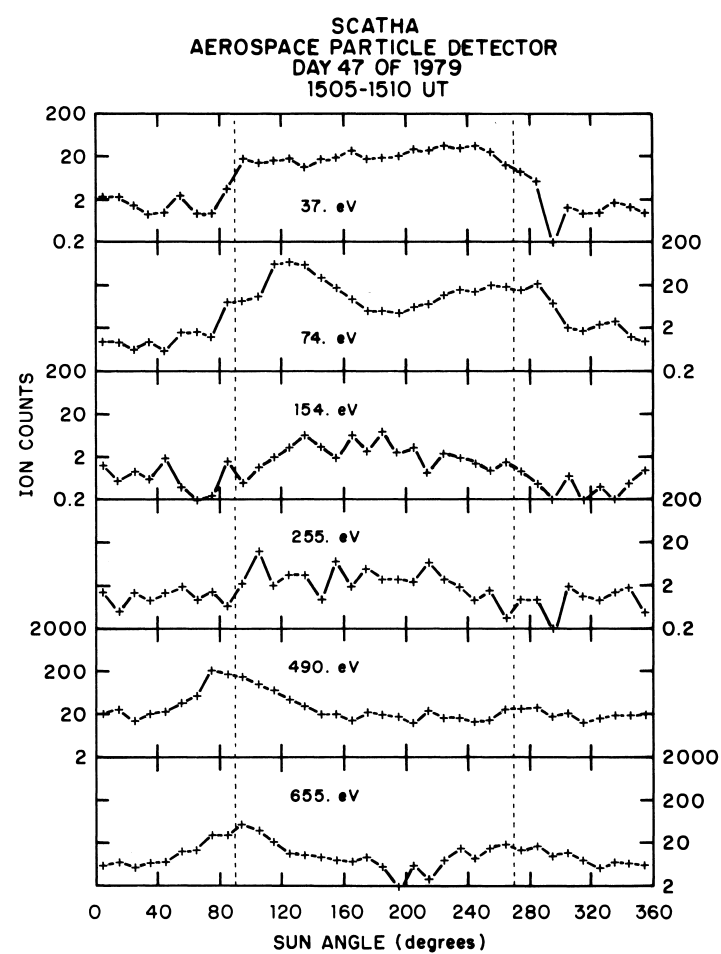

Fig. 6. Ion flux is plotted as a function of sun angle for data accumulated over a 5 minute period on day 47 of 1979. Data are shown from 6 energy channels. The $18 \mathrm{eV}$ channel remained near background. The two top panels show the angular dependence of the spacecraft generated ions. The bottom two panels shows the effects of pitch angle modulation for the ambient (field-aligned) ions. The $490 \mathrm{eV}$ ion flux coincides with the satellite charging peak.

\subsection{Yield}

Theory The calculation of yield due to sputtering is difficult, due to the paucity of good measurements and the lack of clear models for sputtering from polyatomic materials. The available theories and data were combined, and extended to the problem of $\mathrm{H}+$ and $\mathrm{O}+$ sputtering of glass $\left(\mathrm{SiO}_{2}\right)$ [Norwood, 1988]. The general outline of the development is briefly presented here, followed by a comparison of the model with our observations. There are two goals in this process. First, matching the peak flux and second, matching the energy spectrum.

Sputtering is defined as the ejection of material from solid surfaces under ion bombardment. Sputtering yield (number of target atoms sputtered per incident ion) depends on the atomic number of the incident ion, and on the incident ion energy. Yield increases with energy to a maximum and then decreases. Typical values for peak yield are $\sim 1.5$ for neon on Aluminum at $10 \mathrm{keV}$, dropping to less than one for $\mathrm{He}+$ on Aluminum, peaking at $1 \mathrm{keV}$ [Townsend et al, 1976]. The yield maximum increases with ion mass, and shifts to higher energies. These observations led to theories based on atomic collision cascades. Such cascades are created by one incident ion impinging on the lattice and transferring energy to other atoms, which in turn participate in collisions. These theories are based on the simple elastic collisions of classical mechanics, and therefore satisfy the observed dependence on atomic number and momentum transfer. There is a correlation to the heat of sublimation which is explained by assuming the effect of some surface binding energy. The maximum in the energy dependence occurs by postulating some optimum energy deposition depth, beyond which the ability of the cascade particles to reach the surface decreases [Townsend, 1976; McCracken, 1975; Winters, 1976].

The most comprehensive and successful of the collision cascade theories is the Sigmund - Thompson theory. The portion developed by Sigmund treats the sputtering yield of an amorphous (mono-atomic) target. Thompson's theory explains the energy distribution of the sputtered atoms [McCracken, 1975; Winters, 1976; Sigmund, 1969; Thompson, 1968]. The details of the development given here are presented by Norwood [1988].

The Thompson theory, initially developed as a complete model for sputtering, is used to obtain the energy and angle dependence of the emitted spectrum [Thompson, 1969]. The chief features are a spectrum which has the form

$$
Y(E, \phi)=\frac{E}{\left(E+U_{o}\right)^{3}} \cos \phi
$$

Here, $\mathrm{U}_{\mathrm{o}}$ is the binding energy, $\varnothing$ the emitted angle. Winters [1976] shows the good correspondence between the observational data and this energy dependence up to approximately $1 \mathrm{keV}$ [McCracken, 1975; Sigmund, 1969]. The emitted spectra generally show a $\cos \phi$ dependence [McCracken, 1975]. This published dependence may, however, be an artifact due to measurement techniques. This was true for secondary electron yield measurements, as noted by Whipple and Parker [1969].

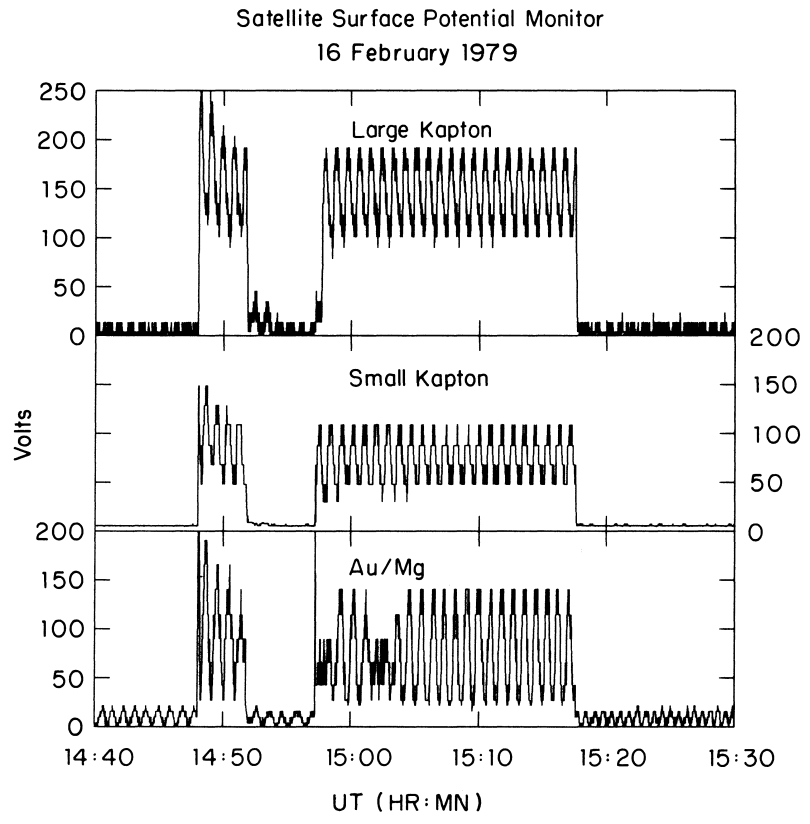

Fig. 7. The potentials for three samples from the SSPM are shown for day 47 of 1979 . The plot corresponds to the period shown in Plate 2. The small and large Kapton samples are on opposite sides of the vehicle, and their potential modulations are $180^{\circ}$ out of phase. 
TABLE 1. Ambient Plasma Values for Days 83, 86, and 200 of 1979

\begin{tabular}{clcc}
\hline Day & Species & Number Density, $\mathrm{cm}^{3}$ & Energy, keV \\
\hline 83 & $\mathrm{H}^{+}$ & 0.38 & 8.9 \\
& $\mathrm{H}_{2}^{+}$ & 0.002 & 120.8 \\
& $\mathrm{He}^{+}$ & 0.003 & \\
86 & $\mathrm{O}^{+}$ & 0.32 & 4.2 \\
& $\mathrm{H}^{+}$ & 0.26 & 5.9 \\
& $\mathrm{H}_{2}^{+}$ & 0.003 & 43.7 \\
& $\mathrm{He}^{+}$ & 0.002 & 3.7 \\
200 & $\mathrm{O}^{+}$ & 0.16 & 4.0 \\
& $\mathrm{H}^{+}$ & 0.91 & 5.3 \\
& $\mathrm{H}_{2}^{+}$ & 0.04 & 7.3 \\
& $\mathrm{He}^{+}$ & 0.22 & 0.4 \\
& $\mathrm{O}^{+}$ & 0.41 & 2.0 \\
\hline
\end{tabular}

Data from the Lockheed ion mass spectrometer.

If we convert the form for yield to distribution function, normalization requirements yield:

$$
Y(E, \phi)=Y \frac{m^{2}}{\pi} \frac{E}{\left(E+U_{o}\right)^{3}} \cos \phi
$$

where $\mathrm{Y}$ is the integral flux, and $\mathrm{m}$ is the mass of the sputtered particle.

Using the Sigmund-Thompson theory, a program was developed to calculate the sputtering yield, incident and emitted flux, and the response of the detector as a function of the energy channel and differential potential, $\Phi$. Data for the ambient plasma compositions was available from the Lockheed Ion Mass Spectrometer for days 83, 86, and 200 of 1979. The values utilized are listed in Table 1.

Calculated Yield The yield obtained in this method was then multiplied by .04 , as approximately $2 \%-4 \%$ of the emitted flux is ionized [D. Harrison, private communication, 1988]. This ion yield is substituted in the count rate integral to obtain the detector responses. The integrals were considered as functions of energy channel and differential potential to determine if the responses could be modeled.

This model can now be applied to the diffuse spectrum observed in the UCSD and Aerospace detectors. We require a broad response, with the particles scattered equally across the energy channels. The diffuse spectrum encountered on day 47 is compared in shape with the model distribution function in Figure 8. An emission angle of $0^{\circ}$ is used. The normalization was reduced until the model overlay the data $(\sim 100 \mathrm{X})$. The distribution function has its maximum in the low energy channels, and compares favorably in shape with data shown for the Fix detector on day 47, from 2-10 eV. The broad spectrum may then be ascribed to the ions returning from nondifferentially charged surfaces. This disagreement in overall normalization is presumably due to a lack of knowledge of the proper $\mathrm{H}+: \mathrm{O}+$ ratio. (Mass spectrometer data are not available).

The goal of matching the narrow (accelerated) distribution was addressed using data from day 86 . The ambient spectra were again used to calculate the sputtered flux, with the goals of matching the narrow energy spectra, and the amplitude of the flux. Figure 9 displays the count rate as a function of energy, using Lo detector data. The goal is to match the five largest energy data points, which represent the locally generated ion population. A differential potential of $28 \mathrm{~V}$ between source and detector was used for the model. Using the observed values for the $\mathrm{O}+$ environment, the model flux is only $\sim 15 \%$ of the observed peak flux. If the yield is multiplied by 6 , the solid

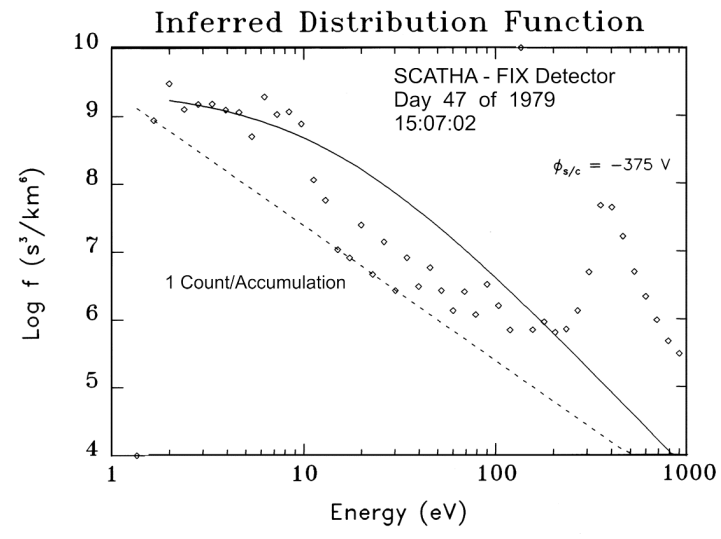

Fig. 8. Day 47 ion distribution function, Fix detector, with modeled emission spectrum. A zero volt differential was assumed between source and detector. Data points are plotted as boxes, the model as a solid line. $\mathrm{H}+/ \mathrm{O}+$ densities and temperatures from Day 86 are used as inputs for the model in the absence of mass spectrometer data for Day 47.

curve results. We see that the model count rate is high over a broader range of energy than the data shown. The data indicate that the flux should be concentrated in only one or two channels. Trajectory effects, treated in the next section, will restrict the allowed energies for each view direction, and should explain the quick dropoff in flux found in Figures 8 and 9. With these caveats, it appears that the model duplicates the observed shape of the ion spectrum, and provides a yield which is within a factor of 10 of the observed flux.

Calculations for the ambient hydrogen population indicate that it can also generate a significant flux at the detectors, simply due to the larger $\mathrm{H}+$ flux. On day 86 , the peak contribution to the yield due to $\mathrm{H}+$ was four counts/s. The relative contributions of these two ambient plasma components vary widely, depending on the temperature and density of their respective distributions. In general, the oxygen contribution is dominant.

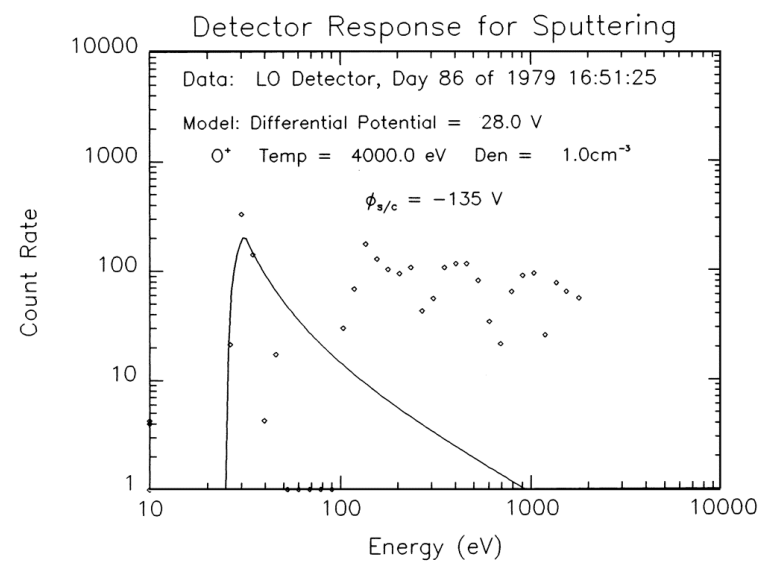

Fig. 9. Modeled ion flux for low energy UCSD detector for O+ incident on silicon, plotted as a solid line. The mass spectrometer inferred density and temperature were used, and a $28 \mathrm{~V}$ potential difference was assumed between the source and detector. Data from the Lo ion detector are superimposed. 
TABLE 2. Modeled and Observed Count Rates

\begin{tabular}{|c|c|c|c|c|}
\hline $\begin{array}{c}\text { Differential } \\
\text { Potential }\end{array}$ & $\underset{\text { Incident }}{\mathrm{H}^{+}}$ & $\begin{array}{c}\mathrm{O}^{+} \\
\text {Incident }\end{array}$ & Total & $\begin{array}{c}\text { Observed } \\
\text { Peak }\end{array}$ \\
\hline \multicolumn{5}{|c|}{ Day 83} \\
\hline 30 & 8 & 74 & 82 & $839^{a}$ \\
\hline 40 & 12 & 115 & 127 & $663^{b}$ \\
\hline 78 & 31 & 283 & 314 & $\begin{array}{r}108^{c} \\
38^{d}\end{array}$ \\
\hline \multicolumn{5}{|c|}{ Day 86} \\
\hline 30 & 5 & 36 & 41 & $\begin{array}{r}329^{e} \\
25^{f}\end{array}$ \\
\hline 100 & 25 & 186 & 211 & $\begin{array}{r}136^{g} \\
25^{h}\end{array}$ \\
\hline \multicolumn{5}{|c|}{ Day 200} \\
\hline 51 & 35 & 117 & 152 & $55^{i}$ \\
\hline 402 & 377 & 1261 & 1638 & $59^{j}$ \\
\hline \multicolumn{5}{|c|}{$\begin{array}{l}{ }^{a} \text { Day 83: Lo detector, } 183^{\circ}, 1755: 08 \mathrm{UT} \text {, peak CR at } 30 \mathrm{eV} . \\
{ }^{b} \text { Day 83: Lo detector, } 165^{\circ}, 1755: 28 \mathrm{UT} \text {, peak CR at } 40 \mathrm{eV} . \\
{ }^{c} \text { Day 83: Lo detector, } 106^{\circ}, 1756: 10 \mathrm{UT} \text {, peak CR at } 78 \mathrm{eV} \text {. } \\
{ }^{d} \text { Day 83: Hi detector, } 92^{\circ}, 1751: 23 \mathrm{UT} \text {, peak CR at } 77 \mathrm{eV} \text {. } \\
{ }^{e} \text { Day 86: Lo detector, } 155^{\circ}, 1651: 25 \mathrm{UT} \text {, peak CR at } 30 \mathrm{eV} \text {. } \\
{ }^{f} \text { Day 86: Hi detector, } 81^{\circ}, 1651: 39 \mathrm{UT} \text {, peak CR at } 30 \mathrm{eV} \text {. } \\
{ }^{g} \text { Day 86: Lo detector, } 100^{\circ}, 1717: 07 \text { UT, peak CR at } 100 \mathrm{eV} \text {. } \\
{ }^{h} \text { Day 86: Hi detector, } 75^{\circ}, 1716: 20 \text { UT, peak CR at } 100 \mathrm{eV} \text {. } \\
{ }^{i} \text { Day 200: Hi detector, } 158^{\circ}, 2208: 31 \text { UT, peak CR at } 51 \mathrm{eV} \text {. } \\
{ }^{j} \text { Day 200: Lo detector, } 156^{\circ}, 2208: 22 \mathrm{UT} \text {, peak CR at } 402 \mathrm{eV} \text {. }\end{array}$} \\
\hline
\end{tabular}

If we compare the peak observed flux with the modeled flux for 3 days analyzed in detail, the values in Table 2 are found (using the mass spectrometer for the ambient $\mathrm{H}+$ and $\mathrm{O}+$ parameters). Flux was measured for various look direction, and hence the energy of the peak and the flux in the peak vary. This variation in peak energy allows a variation in accelerating voltage. For example, on day 83 measurements from the Lo detector at angles from $106^{\circ}-183^{\circ}$ are associated with peak energies from 78-30 eV, and fluxes from 108-839 count/sec. The modeled flux varies from $314-82 \mathrm{c} / \mathrm{s}$. The predicted flux is typically within one order of magnitude of the observations. Given the poor knowledge of sputtering yield, this is not unreasonable. More bothersome is the opposing trends with differential potential. Theory predicts count rate should increase with energy, due to the increased energy window $(\Delta \mathrm{E})$. Observations shows the opposite trend. This discrepancy indicates the larger energy window is not being filled, perhaps because not all trajectories for the measurement correspond to a differentially charged surface. The assumed energy dependence of the emitted spectrum seems to be too broad, also. If the spectrum were slightly narrower, the model curves in Figures 8 and 9 would better represent the data. It is now necessary to consider trajectory effects.

\subsection{Trajectories}

The trajectories to the detectors can be recreated by the NASA Charging Analyzer Program (NASCAP), which was developed to accurately model the dynamics of spacecraft response to realistic plasma environments. NASCAP can effectively simulate the charging, in both laboratory and magnetosphere environments of objects that are geometrically, materially, and electrically complex. The program utilized a time step procedure, calculating quasistatic steps. The dynamics are driven by charge accumulation from external sources, charge depletion, and conduction in dielectrics. Each timestep includes a full three dimensional electrostatic potential calculation. The calculation of LaPlace's equation over a grid is time-staggered with a procedure in which incident charged particle fluxes, leakage currents, emission currents, and induced space charge effects are computed based on the current quasistatic conditions. NASCAP in limited to situations where the Debye length is long compared to the dimensions of the examined object. This condition is met for the SCATHA spacecraft. NASCAP can output a time history of spacecraft charging, potential contours, charge contours, current contours, and particle trajectories. Only the Laplace solver and trajectory algorithms were used here [Katz et al, 1986].

Modeling of the observed angles and energies for the Lo detector was done for the conditions encountered on day 83. A satellite potential of $-250 \mathrm{~V}$ was established, and the resulting potential distribution calculated. Differential potentials were not imposed, nor allowed to develop since we have little knowledge of the actual voltages.

Figure 10 shows model trajectories for the $22.8-115 \mathrm{eV}$ energy bins of the Lo detector. The lowest energy trajectories return very close to the detector. The $155 \mathrm{eV}$ channel corresponds to the last trajectory which hits the solar array panels, for a detector view angle which is 300 below horizontal. This figure is typical of all modeling results for the Lo detector - indications are that the source(s) are close to the detector, on the side of the vehicle (on the solar array). Modeling of the angle-energy pairs indicated in Table 2 produced trajectories which were constrained to the one or two grid cells adjacent to the detectors. (A grid cell is equal in size to the small detector box). The resolution of the code limits our ability to localize the source more closely.

Further modeling of the observations with NASCAP showed that for the $\mathrm{Hi}$ detector (along i) the trajectories generally led back to the top of the satellite. This is illustrated by Figure 11 which shows trajectories for the Hi detector. The trajectories all return to the top of the satellite For the consistently appearing shadow peak, this implies a surface which consistently charges a few tens of volts positive with respect to the satellite. The two main possibilities are the base and boom of the S-band antenna, and the SSPM experiment.

\section{CONCLUSIONS}

The electrostatic analyzer ion measurements from the UCSD and Aerospace detectors both indicate the presence of ions generated on or near the satellite surface during negative charging events. Two distinct classes of behavior are indicated; a diffuse low energy spectrum, and a monoenergetic peak which tracks the satellite potential. The latter spectrum appears to be associated with a source which is differentially charged with respect to the detectors (and satellite frame). The SSPM data indicate differential charging of the proper magnitude can be expected on the satellite insulating surfaces, at least during the induced charging events.

It is reasonable to conclude that sputtering is a physical process which can explain the observed phenomena. It can satisfactorily explain the flux and the energy-angle structure of the triangle and shadow peaks. It also can account for the observed broad spectrum of ions. Detailed agreement is lacking, however. This would require better theories for sputtering yield, a higher grid resolution in the particle tracker, and detailed knowledge of the surface materials. 


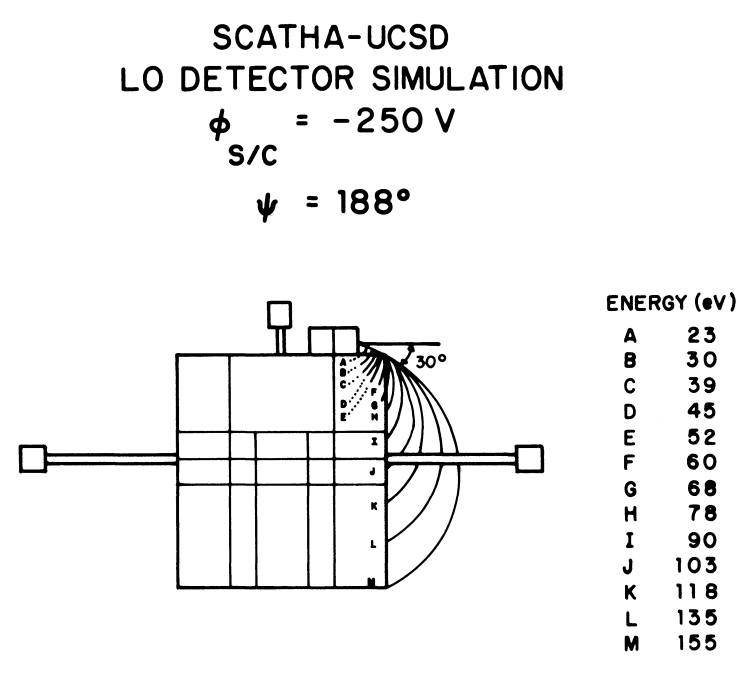

Fig. 10. Trajectory calculations for the Lo ion detector using NASCAP.
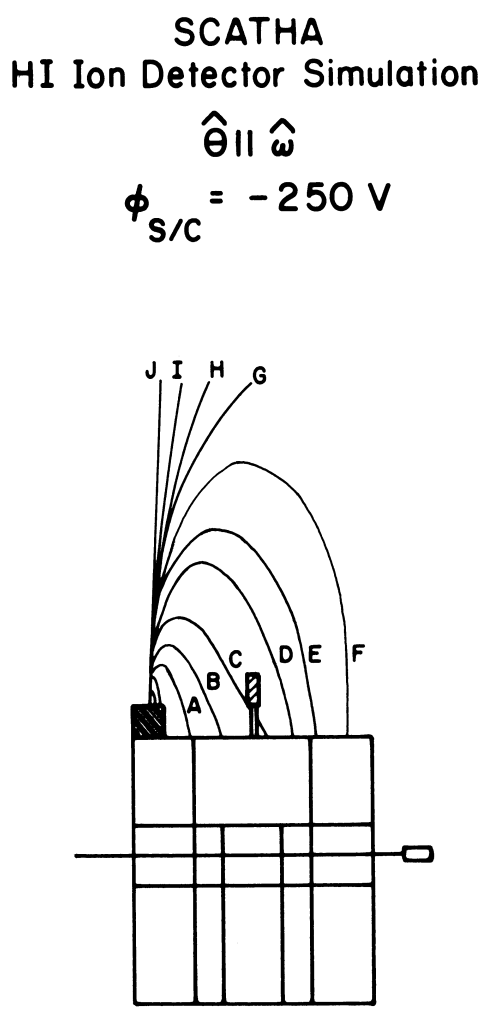

Detector

Energy

$74 \cdot \mathrm{V}$

$91 \bullet V$

$113 \bullet V$

$139 \bullet \mathrm{V}$

$153 \bullet \mathrm{V}$

$173 \bullet V$

$195 \bullet V$

$214 \bullet V$

$264 \bullet V$

$500 \mathrm{eV}$

Fig. 11. Trajectory calculations for the Hi detector.
Mass spectrometer analysis of the low energy ions was not possible on SCATHA. Previously published ISEE 1 data indicated that the mass was greater than $16 \mathrm{AMU}$ [Olsen and Whipple, 1988). These data would be consistent with mass 28silicon. In general, the sputtered ions will not contaminate ion measurements on a positively charged spacecraft.

Since the energy spectrum of the sputtered particles dies off quite rapidly, and the observed differential charges are usually not large, it is not likely that any great contamination of the charging peak results. It may be that outgassing contaminates the charging peak, however. These processes should be reconsidered when the next set of ion mass spectrometer results are available from a charged satellite.

Finally, this work suggests that damage to surface conducting coatings on spacecraft (e.g. ITO) may be a significant problem. These coatings are only a few atoms thick and may be expected to sputter from the surface within several months of the satellite launch. This may help account for the observed charging on the ISEE-1 satellite. This effect is important for those using ion instruments to measure low energy ions on uncharged vehicles, since it represents a possible contamination effect.

Acknowledgements The UCSD detectors were developed by S. DeForest. J. Fennell and D. Croley provided the Aerospace particle data. P. Mizera was the PI for the SSPM. J. Roeder and H. Koons provided the SSPM data. W. W. Li did some of the early work on the UCSD data. D. Harrison and R. Smith helped us develop and apply the sputtering model. This work was supported by the Research Council of the Naval Postgraduate School.

\section{REFERENCES}

Cauffman, D.P., Ionization and Attraction of Neutral Molecules to a Charged Spacecraft, Aerospace Report No. Tr0074(9260-09)-1, Air Force Report . SAMSO-TR-73-263, The Aerospace Corporation, Aug 9. 1973.

DeForest, S.E., Spacecraft charging at synchronous orbit, $J$ Geophys Res, 77, 651-659, 1972.

DeForest, S.E., Electrostatic potentials developed by ATS-5, Photon and Particle Interactions with Surfaces in Space, ed. R.J.L. Grard, 263-276, D,Reidel, Dordrecht, Holland, 1973.

Fennell, J.F., Description of P78-2 (SCATHA) satellite and experiment, pages 68-81, in The IMS Source Book, Guide to the International Magnetospheric Study Data Analysis, edited by C.T. Russell and D.J. Southwood, American Geophysical Union, Wash. DC, 1982.

Katz, I., M. Mandell, G. Jongeward, and M.S. Gussenhoven, The importance of accurate secondary yields in modeling spacecraft charging, J. Geophys. Res., 91, 13739-13744, 1986.

Masek, T.D., and H. Cohen, Satellite positive-ion-beam system, J Space Rockets, 15, 27, 1978.

McCracken, G.M., The behavior of surfaces under ion bombardment, Reports on Progress in Physics, 38. 241, 1975.

Mizera, P.F., Charging results from the Satellite Surface Potential Monitor, J. Space Rockets, 18, 506-509, 1981. 
Mizera, P.F., A summary of spacecraft charging results, $J$. Space Rockets, 20, 438-443, 1983.

Mullen, E.G., M.S. Gussenhoven, D.A. Hardy, T.A.Aggson, B.G. Ledley, E.C. Whipple, SCATHA Survey of highlevel spacecraft charging in sunlight, J.Geophys Res, 91 1474-1490, 1986.

Norwood, C. W., Ions generated on or near satellite surfaces, M.S. Thesis, Naval Postgraduate School, 1988.

Olsen, R.C., C. E. McIlwain, and E. C. Whipple, Observations of differential charging effects on ATS-6, J Geophys Res, 86, 6809-6819, 1981.

Olsen, R.C., and C.K. Purvis, Observations of charging dynamics, J. Geophys Res, 88, 5657-5667, 1983.

Olsen, R.C., and E. C. Whipple, An unusual charging event on ISEE-1, J Geophys Res, 93, 5568-5578, 1988.

Scialdone, J.J., An Estimate of the outgassing of space payloads and its gaseous influence on the environment, J Space Rockets, 23, 373, 1986.

Sigmund, P., Theory of sputtering.I., Phys Rev, 184, 383, 1969.

Thompson, M.W., II. The energy spectrum of atoms during the high energy sputtering of gold, Philosonhical Magazine, 18, 377, 1968.

Townsend, P.D., J.C. Kelly, and N.E.W. Hartley, Ion Implantation, Sputtering, and their Applications, Academic Press, London, 1976, pp. 111-145.

Werner, P.W., Ion Gun Operations at High Altitudes, M.S. Thesis, Naval Postgraduate School, Monterey, CA. June 1988.

Whipple, E. C., and L. W. Parker, Effects on secondary electro emission on electron trap measurements in the magnetosphere and solar wind, J. Geophys. Res., 14, 57635774, 1969.

Winters, H.F., Physical sputtering, Radiation Effects on Solid Surfaces, ed. M.Kaminsky, American Chemical Society, Washington, D.C., 1976. 